\title{
The Resolved Properties of Extragalactic Giant Molecular Clouds
}

\author{
Alberto D. Bolatto ${ }^{1}$, Adam K. Leroy ${ }^{2}$, Erik Rosolowsky ${ }^{3}$, Fabian \\ Walter $^{2}$, and Leo Blitz ${ }^{4}$ \\ ${ }^{1}$ Department of Astronomy, University of Maryland, College Park, MD 20742, USA \\ email: bolatto@astro.umd.edu \\ ${ }^{2}$ Max-Planck-Institut für Astronomie, D-69117 Heidelberg, Germany \\ ${ }^{3}$ Department of Mathematics, Statistics, and Physics, University of British Columbia at \\ Okanagan, Kelowna, B.C. V1V 1V7, Canada \\ ${ }^{4}$ Department of Astronomy, University of California, Berkeley, CA94720, US A
}

\begin{abstract}
Giant molecular clouds (GMCs) are the major reservoirs of molecular gas in galaxies, and the starting point for star formation. As such, their properties play a key role in setting the initial conditions for the formation of stars. We present a comprehensive combined inteferometric/single-dish study of the resolved GMC properties in a number of extragalactic systems, including both normal and dwarf galaxies. We find that the extragalactic GMC properties measured across a wide range of environments, characterized by the Larson relations, are to first order remarkably compatible with those in the Milky Way. Using these data to investigate trends due to galaxy metallicity, we find that: 1) these measurements are not in accord with simple expectations from photoionization-regulated star formation theory; 2) there is no trend in the virial $\mathrm{CO}-$ to- $\mathrm{H}_{2}$ conversion factor on the spatial scales studied; and 3) there are measurable departures from the Galactic Larson relations in the Small Magellanic Cloud - the object with the lowest metallicity in the sample - where GMCs have velocity dispersions that are too small for their sizes. We will discuss the stability of these clouds in the light of our recent far-infrared analysis of this galaxy, and will contrast the results of the virial and far-infrared studies on the issue of the CO-to- $\mathrm{H}_{2}$ conversion factor and what they tell us about the structure of molecular clouds in primitive galaxies.
\end{abstract}

Keywords. ISM: clouds, galaxies: ISM, galaxies: dwarf

\section{Introduction}

There is an emerging body of evidence suggesting that the formation of Giant Molecular Clouds (GMCs) provides the regulating step in transforming gas into stars in galaxies (Leroy et al. 2008; see also Elias Brinks's contribution in these proceedings). Moreover, GMC properties set the initial conditions for protostellar collapse, and likely play a determining role in setting the initial stellar mass function (McKee \& Ostriker 2007). However, there is a dearth of data on the properties of GMCs in other galaxies, particularly low metallicity galaxies. Here we introduce a systematic study of GMCs across dwarf galaxies, and compare their properties with those measured in large galaxies and in the Milky Way. The full analysis is discussed by Bolatto et al. (2008).

Studies of GMCs in the Milky Way (Solomon et al. 1987; Heyer et al. 2004) find that GMCs are in approximate virial equilibrium, and obey uniform scaling relations commonly known as Larson laws (Larson 1981). These originate in the compressible supersonic magnetohydrodynamic turbulence (also known as Burger's turbulence) observed in the interstellar medium (Elmegreen \& Scalo 2004). 


\section{Results and Discussion}

How do extragalactic GMCs compare with those in the Galaxy? We have conducted a systematic study of spatially resolved extragalactic GMCs in galaxies in the Local Group and beyond, using a combination of interferometric and (for the Magellanic Clouds) single-dish CO observations. All observations have been analyzed in the same manner, using the CPROPS algorithm described by Rosolowsky \& Leroy (2007).

Overall, we observe that the relationship between size, velocity dispersion, and luminosity observed for extragalactic GMCs in our sample are consistent with those determined in the Milky Way (Figure 1). This result underscores that the Galactic Larson relations provide a remarkably good description of CO-bright Giant Molecular Clouds independent of their environment, at least in the range of environments explored by this study (our lowest metallicity galaxy, as well as the lowest metallicity galaxy in which CO emission has been reliably detected, is the Small Magellanic Cloud).

Although the Larson relations are approximately Galactic there are some significant departures. GMCs in dwarf galaxies tend to be slightly larger than GMCs in the Milky Way, M 31, or M 33 for a given CO luminosity or velocity dispersion. The largest departures occur in the SMC. GMCs in dwarf galxies have average surface densities that are typically a factor of two under those of the Galaxy $\left(\Sigma_{\mathrm{GMC}} \approx 170 \mathrm{M} \odot \mathrm{pc}^{-2}\right.$ for the Milky Way; Solomon et al. 1987).

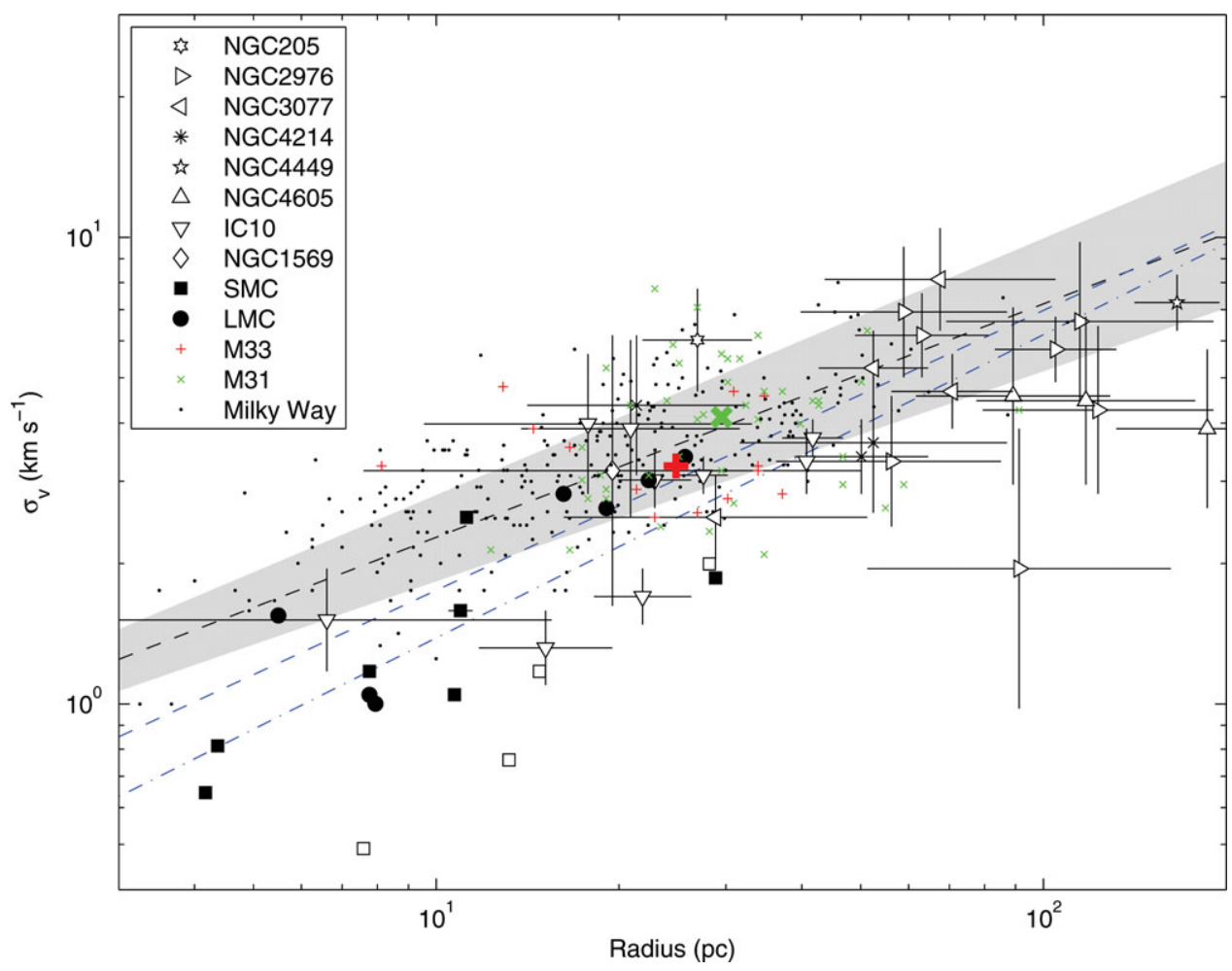

Figure 1. Extragalactic size-line width relation. The gray region as well as the small black points correspond to Solomon et al. (1987). The measurements for each galaxy are shown with the corresponding error bars. For the Magellanic Clouds, white and black symbols correspond to CO $1-0$ and CO 2-1 measurements respectively. The large symbols for M31 and M33 correspond to their averages. The blue dashed and dot-dashed lines illustrate fits to all galaxies and dwarf galaxies only, respectively (see Bolatto et al. 2008 for details). 
In the case of the Small Magellanic Cloud and probably also for IC 10, however, it seems that this explanation is not entirely viable since the departures are much too large and central cloud extinctions would be much too low. We suggest this is indirect evidence for large molecular envelopes faint in $\mathrm{CO}$. We will come back to this point in a moment.

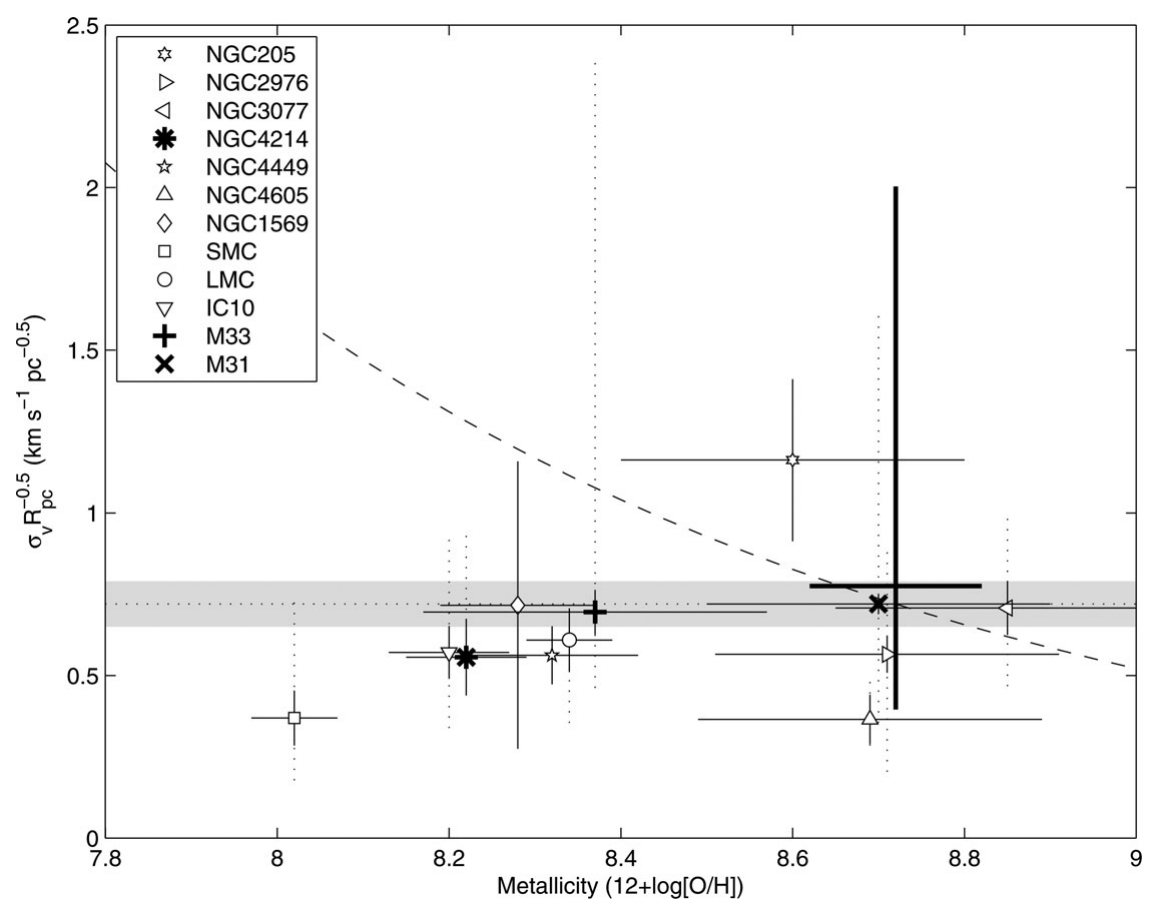

Figure 2. Galaxy averages of surface density vs. metallicity for GMCs in our sample. Filled lines indicate mean uncertainty, dotted lines indicate full observed range. The thick lines correspond to the Milky Way (full range shown in the vertical). The dashed line shows the expectation from photoinization-regulated star formation theory (McKee 1989).

Figure 2 shows that our data do not show evidence for the increase in GMC surface density with decreasing metallicity predicted by photoionization-regulated star formation theory (McKee 1989). Figure 3 shows that we do not see a systematic clear increase in the ratio of the virial to luminous mass (defined as the molecular mass obtained by using the Galactic CO-to- $\mathrm{H}_{2}$ conversion factor) as a function of metallicity in our galaxies. In other words, we see an approximately constant CO-to- $\mathrm{H}_{2}$ conversion factor in these GMCs. In fact, in the range of environments probed (that is, normal and dwarf non-starburst galaxies) the properties of resolved CO-bright GMCs are very uniform. The departure of the Small Magellanic Cloud from the Galactic ratio in Figure 3, for example, is entirely ascribable to the fact that the GMCs in this galaxy tend to be considerably smaller than GMCs in the Milky Way and the relation between virial and luminous mass is weakly dependent on mass (Solomon et al. 1987).

These results stand in contrast to analyses that use the far-infrared (either dust continuum or [CII] line emission) to trace molecular gas in low metallicity environments (e.g., Madden et al. 1997; Leroy et al. 2007). Studies of that type find a large increase in the CO-to- $\mathrm{H}_{2}$ conversion factor and large parcels of CO-faint molecular gas. We suggest that the far-infrared and $\mathrm{CO}$ observations can be simultaneously understood if we assume that bright $\mathrm{CO}$ emission is only associated with the density peaks in metal-poor environments. Observations that resolve these density peaks find, as we show here, prop- 


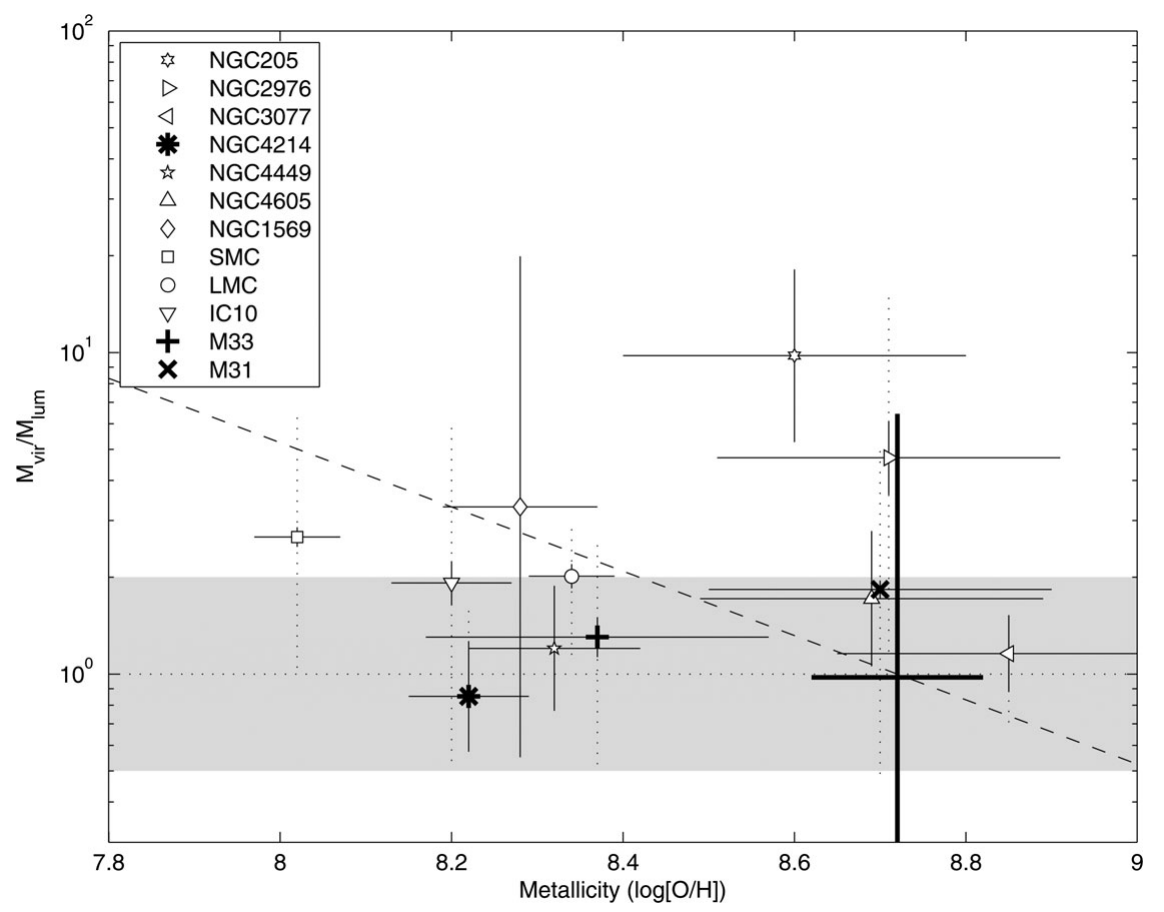

Figure 3. CO-to- $\mathrm{H}_{2}$ conversion factor, obtained as the ratio of virial over luminous mass, versus metallicity for different galaxies in our sample. The gray line indicates the range in the Milky Way. The dashed line is the naive expectation $Z^{-1}$. Error bars are as described in Figure 2.

erties similar to Milky Way GMCs (see Heyer \& Brunt 2004). They miss, however, large envelopes of CO-faint molecular gas. These envelopes would be CO-bright in objects of higher metallicity.

Such envelopes may contain much of the molecular gas as low metallicities (e.g., Leroy et al. 2007). Do they participate in star-formation? In the case of the Small Magellanic Cloud, for example, that appears necessary to preserve a normal star formation efficiency. It is unclear, however, how translucent gas may collapse and give rise to stars. Far-infrared observations of spectral transitions as well as continuum with high spatial resolution will be invaluable to understand the structure of the molecular gas in these enviroments.

\section{References}

Bolatto, A. D., Leroy, A. K., Rosolowsky, E., Walter, F., \& Blitz, L. 2008, ApJ, in the press (arXiv0807.0009)

Elmegreen, B. G. \& Scalo, J. 2004, ARA\&A, 42, 211

Heyer, M. H. \& Brunt, C. M., ApJ, 615, L45

Larson, R. B. 1981, MNRAS, 194, 809

Leroy, A., Bolatto, A., Stanimirovic, S., Mizuno, N., Israel, F., \& Bot, C. 2007, ApJ, 658, 1027

Leroy, A., Walter, F., Brinks, E., Bigiel, F., de Block, W. J. G., Madore, B., \& Thornley, M. D. $2008, A J$, in the press

Madden, S. C., Poglitsch, A., Geis, N., Stacey, G. J., \& Townes, C. H. 1997, AJ, 483, 200

McKee, C. F. 1989, ApJ, 345, 782

McKee, C. F. \& Ostriker, E. C. 2007, ARA\& $\&$, 45, 565

Rosolowsky, E. \& Leroy, A. 2006, PASP, 118, 590

Solomon, P. M., Rivolo, A. R., Barrett, J., \& Yahil, A. 1987, ApJ, 319, 730 